\title{
High grade B-cell lymphoma developed in the course of chronic myeloid leukemia treatment with bosutinib
}

\author{
Teruhito Takakuwa ${ }^{1}$, Ryota Sakai ${ }^{1}$, Shiro Koh ${ }^{1}$, Hiroshi Okamura ${ }^{1}$, Satoru Nanno ${ }^{1}$, \\ Yasuhiro Nakashima ${ }^{1}$, Takahiko Nakane ${ }^{1}$, Hideo Koh ${ }^{1}$, Masayuki Hino ${ }^{1}$, and Hirohisa \\ Nakamae $^{2}$ \\ ${ }^{1}$ Osaka City University Graduate School of Medicine School of Medicine \\ ${ }^{2}$ Osaka City University Graduate School of Medicine
}

May 27, 2020

\begin{abstract}
The 75-year-old male patient had been diagnosed with CML 25 years ago. Over 3 years after starting bosutinib, he was diagnosed with a HGBCL. A total of six courses of DA-EPOCH-R therapy brought complete remission of the lymphoma. Eight months after stopping bosutinib, BCR-ABL1 transcript copies remained undetectable by RT-PCR.
\end{abstract}

\section{Key Clinical Message}

The risk of secondary malignancies due to TKIs is a growing concern. There remains no consensus on the management of secondary lymphoma during TKI therapies. The only alternative is to observe patients receiving TKI treatment cautiously and to treat in the same manner as de novo lymphoma.

\section{Introduction}

Chronic myeloid leukemia (CML) accounts for approximately $15-20 \%$ of all leukemias in adults ${ }^{1}$. CML is characterized by the BCR-ABL1 fusion gene encoding a constitutively active tyrosine kinase ${ }^{2}$. Tyrosine kinase inhibitor (TKI) can help to increase the survival time in CML patients; however, the risk of secondary malignancies due to TKIs is a growing concern. It is reported that second malignancies developed in 3.1$4.5 \%$ of case during the treatment course of CML, of which secondary lymphoma accounts for about $5 \%^{3-5}$. However, few reports showed clinical course of patients who developed lymphoma during TKI therapies. Herein, we report a case of high grade B-cell lymphoma (HGBCL) diagnosed in the course of CML treatment with bosutinib and present the review of literature.

\section{Case}

The 75-year-old male patient was diagnosed with CML 25 years ago (in August 1994) and he started a treatment with interferon. Twelve years later, the patient was started on imatinib. In October 2009, he gradually developed cytopenia. Although there were approximately $3 \%$ blasts in the bone marrow, cytogenetic analysis revealed double $\mathrm{Ph}$ clones, Therefore, the patient was diagnosed with an accelerated phase of CML and the treatment was switched to nilotinib. A cytogenetic response was achieved 3 months after starting treatment with nilotinib, and a major molecular response (MMR) was achieved 2 years after starting nilotinib dosing. The patient developed erythema on the extremities and trunk from the start of nilotinib dosing and antihistamines were continuously administered; however, because the eruptions became uncontrollable, the treatment was changed to bosutinib in March 2016. The MMR was maintained even after switching to bosutinib. 
In early August 2019, the patient developed a posterior neck pain and malaise and was seen at a local medical institution. Computed tomography (CT) revealed lymphadenopathies in the bilateral cervical, mediastinal, and gastric cardial regions, and also around the pancreas head and bilateral inguinal regions. Positron emission tomography showed abnormal accumulation of fluorodeoxyglucose at these same sites (Figure 1). Pathological examinations of the inguinal lymph node biopsies showed cells with large nuclei, proliferating in a starry sky pattern, and immunostaining revealed CD19(+), CD20(+), CD79a $(+)$, MUM1(+), BCL-2(+), c-myc $(+)$, strongly positive Ki-67, CD10(-), TdT(-), and EBER(-). There was no bone marrow infiltration and the patient was diagnosed with a stage III HGBCL. Administration of bosutinib was discontinued since BCR-ABL1 transcript copies remained below the level of detection achieved by the real-time quantitative reverse transcription polymerase chain reaction (RT-PCR). After two courses of the dose-adjusted EPOCH$\mathrm{R}$ (etoposide, prednisone, vincristine, cyclophosphamide, doxorubicin, and rituximab) therapy, a complete remission (CR) was confirmed on $\mathrm{CT}$ scan. The $\mathrm{CR}$ was also maintained after 4 additional courses (a total of 6 courses) of the same therapy. Moreover, BCR-ABL1 transcript copies remained undetectable by RT-PCR 8 months after bosutinib discontinuation.

\section{Discussion}

The present patient developed lymphoma after being treatment with three different TKIs. The patient developed HGBCL after administration of bosutinib. This is a valuable case because no previous studies have reported the detailed clinical course of such a case.

The risk of secondary cancer due to TKI has been a subject of debate. Novartis reported that among 9,518 CML patients administered imatinib, the incidence of secondary malignancy was $1.16 \%$, and that cancer onset did not increase by administration of imatinib ${ }^{6}$. This data, however, is based on spontaneous reports and it is possible that secondary cancers were under-reported. MD Anderson Cancer Center also reported that TKI does not increase the risk of cancer $^{7}$, but their data included patients with diseases other than CML. Contrarily, albeit from a small sample size, Roy L et al. observed secondary cancer in $3.17 \%$ of patients after 8-36 months of imatinib administration, and reported a particularly high risk of prostate cancer among them ${ }^{3}$. Shah BK et al. reported that the risk of cancer onset in CML before the introduction of imatinib was equal to that of healthy individuals, but that the risk of secondary malignancies in CML has increased since its introduction ${ }^{8}$. Sweden has also reported that the risk of cancer in CML patients who were administered TKI increases by approximately 1.5 -fold that of the general population ${ }^{9}$. In a survey of 13,256 CML patients, Sasaki K et al. reported a $4.5 \%$ incidence of secondary malignancies ${ }^{4}$. In this study, patients who had a history of cancer at the time of CML diagnosis and those who developed other cancers within one year of the CML diagnosis were excluded from this report. Furthermore, the 10-year risk of secondary malignancy was stable. These reports suggest that TKI may increase the risk of secondary malignancies and it is therefore necessary to cautiously observe patients receiving a TKI treatment for the onset of other cancers.

Sasaki K et al. reported $31(0.2 \%)$ CML patients with secondary lymphoma, but their clinical courses are unknown. Table 1 showed the detailed clinical course of lymphomas that developed during TKI treatment in CML patients ${ }^{10-17}$. Among them, imatinib was the most commonly used medication, while there were no reports of patients who were treated with bosutinib. The time from the initial TKI dose to the onset of the secondary cancer was longest in our case. The outcomes of the lymphoma are unknown for case 4 and 8 , while case 3 died 8 months after developing the lymphoma. Other cases responded well to chemotherapy.

Several possible mechanisms of the onset of secondary cancer in CML patients under TKI therapy may be thought possible. First, CML itself may increase the risk of cancer onset. It is possible that BCR-ABL translocation may introduce genetic instability and that progenitors of solid cancers or other hematological malignancies were already latent at the time of CML diagnosis ${ }^{18}$. However, patients who had been in longterm remission have also been observed with secondary cancer; therefore, this hypothesis alone does not fully explain the mechanisms of onset. Second, it is possible that TKI is oncogenic. In vitro studies have reported that imatinib induces irreversible chromosomal abnormalities or aberrations ${ }^{19}$. This chromosome instability may influence the development of the earliest stages of cancer ${ }^{20}$. Third, and possibly most importantly, 
TKI-induced immunosuppression may leave patients vulnerable to secondary cancers. Generally, patients with compromised immune systems are more prone to develop malignancies ${ }^{21}$. TKIs are known to inhibit the proliferation or function of T cells, B cells, and NK cells ${ }^{22}$, and this may decrease tumor immunity, thereby contributing to the cancer onset. Thus, as long as patients are on TKI therapy, they must be considered as being exposed to the risk of secondary malignancies. Although Epstein-Barr virus can contribute to the pathogenesis of lymphoma, particularly in compromised patients ${ }^{23}$, Epstein-Barr encoding region in situ hybridization was negative with this patient's specimens.

There are no clear reports on the prognosis of lymphoma developed during TKI therapy. The present patient responded rapidly to initial therapy as well as most of other patients displayed in Table 1. Lymphoma arising in patients with primary immunodeficiencies is generally known to have poor a prognosis ${ }^{24}$. However, the prognosis of most of iatrogenic immunodeficiency-associated lymphomas is not poor ${ }^{25}$. Presently, the only treatment available for lymphomas resulting from TKI therapy is the same as that for de novo lymphoma. Accumulating data from more patients is needed for the development of novel therapeutic strategies for lymphomas secondary to TKI.

\section{Author Contribution}

Teruhito Takakuwa wrote the manuscript with support from Hirohisa Nakamae. Ryota Sakai designed a figure and a table. All authors discussed the case and contributed to the final manuscript.

\section{References}

1. Siegel RL, Miller KD, Jemal A. Cancer Statistics, 2017. CA: a cancer journal for clinicians . Jan 2017;67(1):7-30. doi:10.3322/caac.21387

2. Sattler M, Griffin JD. Mechanisms of transformation by the BCR/ABL oncogene. International journal of hematology . Apr 2001;73(3):278-91. doi:10.1007/bf02981952

3. Roy L, Guilhot J, Martineau G, Larchee R, Guilhot F. Unexpected occurrence of second malignancies in patients treated with interferon followed by imatinib mesylate for chronic myelogenous leukemia.Leukemia . Sep 2005;19(9):1689-92. doi:10.1038/sj.leu.2403874

4. Sasaki K, Kantarjian HM, O'Brien S, et al. Incidence of second malignancies in patients with chronic myeloid leukemia in the era of tyrosine kinase inhibitors. International journal of hematology . May 2019;109(5):545-552. doi:10.1007/s12185-019-02620-2

5. Krishnan B, Morgan GJ. Non-Hodgkin lymphoma secondary to cancer chemotherapy. Cancer epidemiology, biomarkers $\& 3$ prevention : a publication of the American Association for Cancer Research, cosponsored by the American Society of Preventive Oncology . Mar 2007;16(3):377-80. doi:10.1158/1055-9965.epi-06-1069

6. Pilot PR, Sablinska K, Owen S, Hatfield A. Epidemiological analysis of second primary malignancies in more than 9500 patients treated with imatinib. Leukemia. Jan 2006;20(1):148; author reply 149. doi:10.1038/sj.leu.2404025

7. Verma D, Kantarjian H, Strom SS, et al. Malignancies occurring during therapy with tyrosine kinase inhibitors (TKIs) for chronic myeloid leukemia (CML) and other hematologic malignancies. Blood . Oct 20 2011;118(16):4353-8. doi:10.1182/blood-2011-06-362889

8. Shah BK, Ghimire KB. Second primary malignancies in chronic myeloid leukemia. Indian journal of hematology 83 blood transfusion : an official journal of Indian Society of Hematology and Blood Transfusion . Dec 2014;30(4):236-40. doi:10.1007/s12288-013-0328-2

9. Gunnarsson N, Stenke L, Hoglund M, et al. Second malignancies following treatment of chronic myeloid leukaemia in the tyrosine kinase inhibitor era. British journal of haematology . Jun 2015;169(5):683-8. doi:10.1111/bjh.13346 
10. Rodler E, Welborn J, Hatcher S, et al. Blastic mantle cell lymphoma developing concurrently in a patient with chronic myelogenous leukemia and a review of the literature. American journal of hematology . Apr 2004;75(4):231-8. doi:10.1002/ajh.20025

11. Mihaylov G, Varbanova V, Stoeva V, Dikov T. Extranodal marginal zone B-cell lymphoma arising in chronic myeloid leukaemia successfully treated with tyrosine kinase inhibitor: a case report.Hippokratia . Jul-Sep 2016;20(3):241-243.

12. Takeyasu Y, Satake A, Azuma Y, et al. Tyrosine kinase inhibitor and rituximab-CHOP treatment for concurrent chronic myeloid leukemia and non-Hodgkin lymphoma: a case report. Clinical case reports . Dec 2017;5(12):2047-2050. doi:10.1002/ccr3.1253

13. Abuelgasim KA, Rehan H, Alsubaie M, et al. Coexistence of chronic myeloid leukemia and diffuse large B-cell lymphoma with antecedent chronic lymphocytic leukemia: a case report and review of the literature. Journal of medical case reports . Mar 11 2018;12(1):64. doi:10.1186/s13256-018-1612-4

14. Fujiwara SI, Shirato Y, Ikeda T, et al. Successful treatment of follicular lymphoma with secondgeneration tyrosine kinase inhibitors administered for coexisting chronic myeloid leukemia.International journal of hematology. Jun 2018;107(6):712-715. doi:10.1007/s12185-017-2378-y

15. Gajendra S, Sharma A, Sharma R, Gupta SK, Sood N, Sachdev R. Hodgkin Lymphoma in a Case of Chronic Myeloid Leukemia Treated with Tyrosine Kinase Inhibitors. Turk patoloji dergisi . 2019;35(1):7478. Hodgkin Lymphoma in a Case of Chronic Myeloid Leukemia Treated with Tyrosine Kinase Inhibitors. doi:10.5146/tjpath.2016.01368

16. Cai Z, Liu S, Zi J, Ma J, Ge Z. A case of primary gastric diffuse large B-cell lymphoma occurring in chronic myeloid leukemia. Onco Targets and therapy . 2019;12:5917-5923. doi:10.2147/ott.s212838

17. Dominguez-Pinilla N, Martinez-Zamorano E, Campos-Martin Y, et al. Paediatric-type nodal follicular lymphoma in a child diagnosed with chronic myeloid leukaemia. British journal of haematology . Sep 2019;186(6):e207-e209. doi:10.1111/bjh.16089

18. Fabarius A, Kalmanti L, Dietz CT, et al. Impact of unbalanced minor route versus major route karyotypes at diagnosis on prognosis of CML.Annals of hematology . Dec 2015;94(12):2015-24. doi:10.1007/s00277-015-2494-9

19. Fabarius A, Giehl M, Frank O, et al. Induction of centrosome and chromosome aberrations by imatinib in vitro. Leukemia . Sep 2005;19(9):1573-8. doi:10.1038/sj.leu.2403861

20. Pihan GA, Wallace J, Zhou Y, Doxsey SJ. Centrosome abnormalities and chromosome instability occur together in pre-invasive carcinomas. Cancer research . Mar 15 2003;63(6):1398-404.

21. Duan L, Grunebaum E. Hematological Malignancies Associated With Primary Immunodeficiency Disorders. Clinical immunology (Orlando, Fla) . Sep 2018;194:46-59. doi:10.1016/j.clim.2018.06.011

22. Haguet H, Douxfils J, Chatelain C, Graux C, Mullier F, Dogne JM. BCR-ABL Tyrosine Kinase Inhibitors: Which Mechanism(s) May Explain the Risk of Thrombosis? TH open : companion journal to thrombosis and haemostasis . Jan 2018;2(1):e68-e88. doi:10.1055/s-0038-1624566

23. Shannon-Lowe C, Rickinson AB, Bell AI. Epstein-Barr virus-associated lymphomas. Philosophical transactions of the Royal Society of London Series B, Biological sciences . Oct 19 2017;372(1732)doi:10.1098/rstb.2016.0271

24. Parvaneh N, Filipovich AH, Borkhardt A. Primary immunodeficiencies predisposed to EpsteinBarr virus-driven haematological diseases.British journal of haematology . Sep 2013;162(5):573-86. doi:10.1111/bjh.12422

25. Tokuhira M, Watanabe R, Nemoto T, et al. Clinicopathological analyses in patients with other iatrogenic immunodeficiency-associated lymphoproliferative diseases and rheumatoid arthritis. Leukemia $\mathscr{E}$ lymphoma 
. Apr 2012;53(4):616-23. doi:10.3109/10428194.2011.625101

Figure.1 PET/CT images during the diagnosis of the high grade B-cell lymphoma.

Fused positron emission tomography showing increased fluorodeoxyglucose uptake at the bilateral cervical and also around the pancreas head and bilateral inguinal regions. 


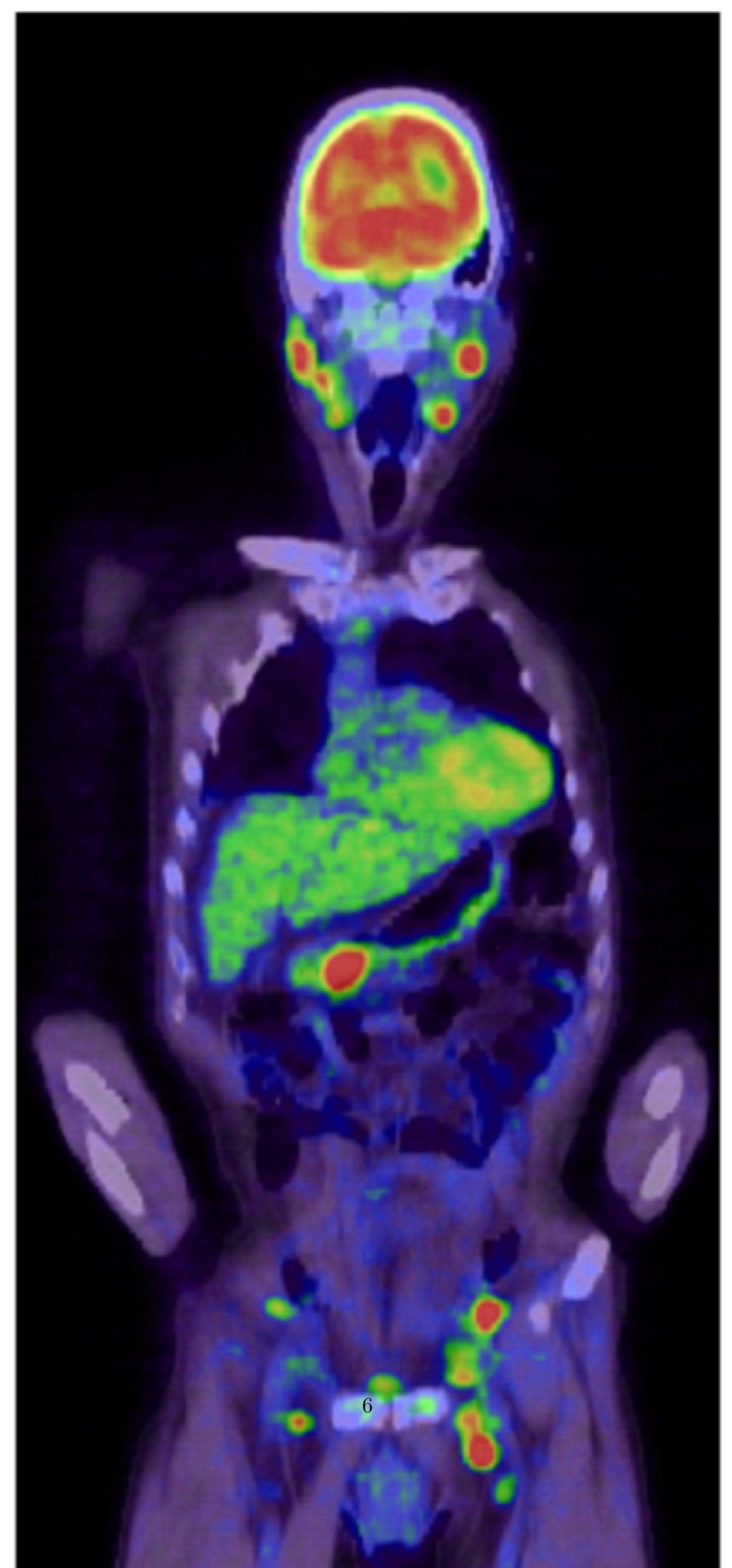




\section{Hosted file}

Table 1.docx available at https://authorea.com/users/326919/articles/454614-high-gradeb-cell-lymphoma-developed-in-the-course-of-chronic-myeloid-leukemia-treatment-withbosutinib 